TRANSACTIONS OF THE

AMERICAN MATHEMATICAL SOCIETY

Volume 188, Issue 2, 1974

\title{
A DISCONTINUOUS INTERTWINING OPERATOR
}

BY

\author{
ALLAN M. SINCLAIR
}

ABSTRACT. If $T$ and $R$ are continuous linear operators on Banach spaces $X$ and $Y$ with the spectrum of $R$ countable, we obtain necessary and sufficient conditions on the pair $T, R$ that imply the continuity of every linear operator $S$ from $X$ in to $Y$ satisfying $S T=R S$.

Introduction. We are concerned with conditions on a pair of continuous linear operators $T$ and $R$ on complex Banach spaces $X$ and $Y$ that imply the continuity of every linear operator $S$ from $X$ into $Y$ intertwining with $T$ and $R$ (i.e. such that $S T=R S$ ). This problem is discussed in [4], [5] and [6]. When $T=R$ is compact or quasinilpotent, necessary and sufficient conditions on $T$ were obtained in [6] (and [S] for quasinilpotent) that imply the continuity of $S$. In Theorem 3.3 of [6] necessary conditions on $T$ and $R$ were obtained for the continuity of $S$ provided the spectrum of $R$ was countable. We show that a slight modification of these conditions is necessary and sufficient for the continuity of $S$ again when the spectrum of $R$ is countable (Theorem 2.2). We also obtain necessary and sufficient conditions on $T$ and $R$ that ensure that $S$ is continuous when $o(T) \cap \sigma(R)$ is empty (Theorem 2.1). This latter result shows that the hypothesis of the continuity of $S$ cannot be omitted from the well-known application of the analytic functional calculus that states that $R, S, T$ continuous, $S T=R S$, and $\sigma(T) \cap \sigma(R)=\varnothing$ implies that $S$ is zero.

The method of proof is similar to that used in [6] and [5]. We shall use the same notation as [6]. The basic idea is to turn $X$ and $Y$ into modules over the principal ideal domain $\mathrm{C}[x]$ of polynomials in an indeterminate $x$ via the operators $T$ and $R$, and use properties of an injective submodule of $Y$.

Recall that a linear subspace $Z$ of $Y$ is $R$-divisible if $(R-\lambda I) Z=Z$ for all complex numbers $\lambda$. If $R$ is a linear operator on $Y$, we regard $Y$ as a $C[x]$-module by defining $p(x) \cdot y=p(R) y$ for all $p$ in $C[x]$ and all $y$ in $Y$. Then an R-divisible subspace is just a divisible $C[x]$-submodule, and by the standard theory of modules over $\mathbf{C}[x]$ this is an injective submodule [2, p. 134]. We shall thus talk of $R$ divisible subspaces when we wish to emphasise the linear operator $R$, and

Received by the editors November 28, 1972 and, in revised form, May 10, 1973. $13 \mathrm{C10}$.

AMS (MOS) subject classifications (1970). Primary 47199; Secondary 47D99, 13J05,

Key words and phrases. Automatic continuity, intertwining operator, divisible subspace, injective $C[x]_{\text {module, countable spectrum. }}$ 
injectives when we wish to lift $\mathbf{C}[x]$-homomorphisms. For convenience we give two definitions that occur in [6]. A complex number $\lambda$ is a critical eigenvalue of $\{T, R\}$ if $(T-\lambda I) X$ is of infinite codimension in $X$ and if $\lambda$ is an eigenvalue of $R$. If $E$ is a subset of the complex numbers, we denote by

$$
\bigcap^{\infty}(\lambda \in E)(R-\lambda I) Y
$$

the eventual constant value of the transfinite sequence defined by $Y(0)=Y$,

$$
Y(a+1)=\bigcap\{(T-\lambda I) Y(a): \lambda \in E\},
$$

and for limit ordinals $a$

$$
Y(\alpha)=\bigcap\{Y(\beta): \beta<\alpha\} \text {. }
$$

The first section contains the existence of a discontinuous $S$ intertwining with $T$ and $R$ under the assumptions that $T$ is not algebraic and $R$ has a nonzero divisible subspace. This result is applied in $\$ 2$. In the third section we show that if $R$ has a nonzero divisible subspace then it has a torsion free divisible subspace; that is, that in the decomposition of this injective into a direct sun terms of the type $\mathrm{C}(x)$ occur. All linear spaces and linear operators are over the complex field.

I am grateful to the referee for suggestions which simplified the proof of Lemma 1.1 and led to a strengthening of Lemma 3.2.

1. A discontinuous intertwining operator. The basic idea behind the proof of Theorem 1.2 is (1) to show that if a continuous linear operator $T$ on a Banach space $X$ is not algebraic then there is an infinitely generated free $\mathbf{C}[x]$-submodule of $X$, and (2) to apply the theory of injective modules to lift a discontinuous linear operator constructed from this free $\mathrm{C}[x]$-module. We shall require the following lemma in $\$ 3$ for a Fréchet space rather than just a Banach space. Recall that a $\mathrm{C}[x]$-module $X$ has an infinitely generated free $\mathrm{C}[x]$-submodule $Z$ if there is an infinite set $U$ in $X$ such that $Z$ is the direct sum of $\mathrm{C}[x] \cdot u$ as $u$ runs over $U$, and each $\mathrm{C}[x] \cdot u$ is isomorphic to $\mathrm{C}[x]$ under the map $p(x) \cdot u \rightarrow p(x)$.

Lemma 1.1. Let $X$ be a Fréchet space, and let $T$ be a continuous linear operator on $X$. If $T$ is not algebraic, then $X$ contains an infinitely generated free $\mathrm{C}[x]$-submodule.

Proof. The proof is an adaptation of $\left[7\right.$, Theore:n 15, p. 40]. Let $X^{\omega}$ be the space of all sequences from $X$ with the product topology, and let $C[x]_{0}$ be the space of all sequences froin $\mathrm{C}[x]$ of polynomials with only a finite number of nonzero entries. If $p$ is in $C[x]_{0}$ and $y$ is in $X^{\omega}$, then we define $P y=\Sigma p_{j} \cdot y_{j}=$ $\Sigma p_{j}(T) y_{j}$.

For $P$ in $C[x]_{0}$ we let $\operatorname{deg} P=\max \left\{\operatorname{deg} p_{j}\right\}$, Supp $P=\left\{j: p_{j} \neq 0\right\}$, and $\|P\|_{1}$ 
$=\Sigma\left|a_{i j}\right|$, where $P=\left(p_{1}, p_{2}, \cdots\right)$, and $p_{j}(x)=\Sigma a_{i j} x^{i}$. For each positive integer $n$ we let

$$
\begin{aligned}
F_{n}=\left\{y \in X^{\omega}: P y=\right. & 0 \text { for some } p \in C[x]_{0} \text { with } \operatorname{deg} P<n, \\
& \text { Supp } \left.P \subseteq\{1, \cdots, n\} \text { and } 2^{-n} \leq\|P\|_{1} \leq 2^{n}\right\} .
\end{aligned}
$$

Then $F_{n}$ is closed for each $n$ because of the compactness of the set of $P$ in $\mathrm{C}[x]_{0}$ that defines $F_{n}$.

If $X$ contains no infinitely generated free $\mathrm{C}[x]$-submodule, then the set $\left\{F_{n}\right\}$ covers $X^{\omega}$. Hence by the Baire category theorem [3, p. 299] there is an $n$ such that $F_{n}$ has nonempty interior. Let $y^{\sim}$ be in the interior of $F_{n}$. Then $F_{n}-y^{\sim}$ is a neighbourhood of 0 in $X^{\omega}$. By homogeneity for each $y$ in $X^{\omega}$ there is a $P$ in $C x]_{0}$ with Supp $P \subseteq\{1, \cdots, n\}$, deg $P<2 n$, and $P y=0$. This $P$ is obtained as the product of the $P^{\sim}$ in $\left.\mathrm{Q} x\right]_{0}$ associated with $y^{\sim}$ and that associated with $\beta y+y^{\sim}$ for some suitably small scalar $\beta$. If $x$ is in $X$, we apply this to $y=\left(x, T^{2 n} x, T^{4 n} x, \cdots\right)$ in $X^{\omega}$. This gives a nonzero polynomial $q$ of degree less than $2(n-1) n^{2}$ satisfying $q(T) x=0$. By $[7$, Lemma 14, p. 41] this implies that $T$ is algebraic, and completes the proof of the lemma.

Theorem 1.2 Let $X$ and $Y$ be Banach spaces, and let $T$ and $R$ be continuous linear operators on $X$ and $Y$, respectively. If $T$ is not algebraic, and if $Y$ bas a nonnull $R$-divisible subspace, then tbere is a discontinuous linear operator $S$ from $X$ into $Y$ sucb that $S T=R S$.

Proof. By Lemma 1.1 there are $z_{1}, z_{2}, \cdots$ in $X$ such that $W=\mathrm{C}[T] z_{1} \oplus$ $\mathrm{C}[T] z_{2} \oplus \ldots$ is a direct sum, and by normalizing $z_{j}$ we may assume that $\left\|z_{j}\right\|=1$. Let $y$ be a nonzero element in an $R$-divisible subspace of $Y$. We define $S_{0}: W \rightarrow$ $Y$ by $S_{0} p(T) z_{j}=j p(R) y$ for each $p$ in $\mathrm{C}[x]$ and each $j$, and by linearity on $W$. Then $S_{0}$ maps $W$ into an $R$-divisible subspace of $Y$, we have $S_{0} T=R S_{0}$ where $T$ is restricted to $W$, and $S_{0}$ is discontinuous. Hence $S_{0}$ is a $C[x]$-homomorphism from a submodule of $X$ into an injective submodule of $Y$, so $S_{0}$ may be extended to a homomorphism $S$ from $X$ into the injective submodule of $Y$. This $S$ is the required linear operator.

2. Automatic continuity of an intertwining operator. In this section we give necessary and sufficient conditions on $T$ and $R$, under additional assumptions on the spectrum of $R$, that imply the automatic continuity of a linear operator $S$ intertwining with $T$ and $R$. The additional restrictions are that $\sigma(T) \cap \sigma(R)$ is empty (Theorem 3.1) and that $\sigma(R)$ is countable (Theorem 2.22). This raises the natural question as to whether the hypothesis $\sigma(R)$ is countable in Theorem 2.2 can be weakened to $\sigma(R) \cap \sigma(T)$ is countable, as such a weakening would contain both Theorems 2.1 and 2.2. 
Theorem 21. Let $X$ and $Y$ be Banach spaces, and let $T$ and $R$ be continuous linear operators on $X$ and $Y$, respectively, with disjoint spectra. Then there is a discontinuous linear operator $S$ satisfying $S T=R S$ if, and only if, $T$ is not algebraic and $Y$ bas a nonnull $R$-divisible subspace.

Proof. By Theorem 1.2 we only have to show that the existence of a discontinuous $S$ implies that $T$ is not algebraic and that $Y$ has a nonnull $R$-divisible subspace. Suppose that $p$ is a polynomial with $p(T)=0$. We may assume that the zeros of the polynomial $p$ are in the spectrum of $T$. Then $\operatorname{Sp}(T)=p(R) S=0$ so that $S=0$, because none of the zeros of $p$ are in the spectrum of $R$. This gives a contradiction.

Let $Y_{\infty}$ denote the maximal $R$-divisible subspace of $Y$. Then $Y_{\infty}=$ $\bigcap^{\infty}(\lambda \in \sigma(R))(R-\lambda l) Y$ as is noted in [6, 2.3(d), p. 534]. If $\lambda$ is in $\sigma(R)$, then $S X=S(T-\lambda l) X=(R-\lambda I) S X$ so that a standard transfinite induction argunent shows that $S X \subseteq Y_{\infty}$. Since $S X \neq\{0\}$, there is a nonnull $R$-divisible subspace of $Y$. This completes the proof.

Our next result gives slightly different conditions to Theorem 3.3 of $[6, \mathrm{p}$. $537]$ but these conditions turn out to be necessary and sufficient unlike those of [6, Theorem 3.3, p. 537].

Theorem 2.2. Let $X$ and $Y$ be Banach spaces, and let $T$ and $R$ be continuous linear operators on $X$ and $Y$, respectively. Suppose that $\sigma(R)$ is countable. Then every linear operator $S$ from $X$ into $Y$ satisfying $S T=R S$ is continuous if, and only if,

(i) $\{T, R\}$ bas no critical eigenvalues, and

(ii) either (a) $T$ is algebraic or (b) $\{0\}$ is the only R-divisible subspace of $Y$.

Proof. If (i) fails to hold, then there is a discontinuous $S$ by [4] or [6, Lemma 2.1, p. 533]. If (ii) fails to hold, then there is a discontinuous $S$ by Theorem 1.2. If (i) and (ii)(b) hold, then the automatic continuity of $S$ is [6, Theorem 3.3, p. 537].

We suppose that (i) and (ii) (a) hold. Then $\sigma(T)$ is finite and equal to $\left\{\lambda_{1}, \cdots, \lambda_{n}\right\}$, say. That $T$ satisfies a polynomial equation and has spectrum $\left\{\lambda_{1}, \cdots, \lambda_{n}\right\}$ implies that there are closed linear subspaces $X_{1}, \cdots, X_{n}$ in $X$ such that $X=X_{1} \oplus \cdots \oplus X_{n}$ and $\left(T-\lambda_{j} I\right)^{r} X_{j}=\{0\}$ for some positive integer $r_{j}$ and $j=1, \cdots, n$. If $\lambda_{j}$ is not an eigenvalue of $R$, then

$$
\left(R-\lambda_{j} l\right)^{r} i S X_{j}=S\left(T-\lambda_{j} l\right)^{r}{ }^{i} X_{j}=\{0\},
$$

so that $S X_{j}=\{0\}$. If $\lambda_{j}$ is an eigenvalue of $R$, then $\left(T-\lambda_{j} D X\right.$ is cofinite in $X$, because $(T, R)$ has no critical eigenvalues, and hence $\left(T-\lambda_{j} l\right)^{{ }^{j}} X$ is cofinite 
in $X$. Since $\left(T-\lambda_{j} I\right) X_{k}=X_{k}$ for $k \neq j$, and $\left(T-\lambda_{j} l\right)^{r}{ }^{j} X_{j}=\{0\}$, we have

$$
\left(T-\lambda_{j} l\right)^{r_{j}} X=X_{1} \oplus \cdots \oplus X_{j-1} \oplus X_{j+1} \oplus \cdots \oplus X_{n} \text {. }
$$

Thus $X_{j}$ is finite dimensional. Therefore $S$ is zero on the direct sum of $X_{k}$ for those $\lambda_{k}$ that are not eigenvalues of $R$, and this direct sum is a closed cofinite subspace of $X$. Hence $S$ is continuous, completing the proof.

3. Torsion free injective submodules. The main result of this section is Theorem 3.3 in which we show that if $Y$ has a nonnull $R$-divisible subspace, then there is a nonnull torsion free $R$-divisible subspace. We shall use this result to show that a discontinuous linear operator $S$ may be chosen under the hypotheses of Theorem 1.2 to be an isomorphism on a singly generated free $\mathrm{C}[x]$-submodule of $X$. The operator $S$ obtained in this way is more complex than the finite rank discontinuous linear operator $S$ constructed in [6, Lemma 2.1, p. 533] and [4, Lemma 2.1, p. 88]. As the construction of Theorem 1.2 stands, the discontinuous $S$ produced could have finite rank. For example, suppose that the $y$ chosen satisfies $R y=0$ and that each $z_{j}$ is not in $T X$. Then the $S$ produced by Theorem 1.2 could have range $C y$ and annihilate $T X$ so making it the discontinuous linear operator of [6, Lemma 2.1], which is given by an extension of linear functionals without any discussion of modules. To prove Theorem 3.3 we use a torsion element in an $R$-divisible subspace of $Y$ to construct a subspace that is isomorphic as a $\mathrm{C}[x]$-module to a weighted sequence space and shift operator. This shift operator is shown to have a torsion free divisible subspace that has an infinite number of direct summands each $C[x]$-isomorphic to $C(x)$, the

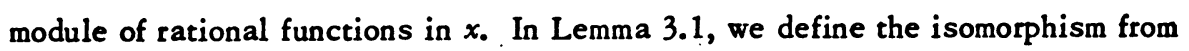
a weighted sequence space into a Banach space, and in Lemma 3.2 check that the shift on this sequence space has the required nonnull torsion free divisible subspace. We denote the set of complex sequences tending to zero by $c_{0}$ and the supremum norm by $\|\cdot\|_{\infty}$.

Lemma 3.1. Let $Y$ be a Banacb space, and let $y_{0}, y_{1}, \cdots$ be linearly independent elements in $Y$. Then there exists a sequence $\alpha_{n}$ that tends to infinity, and if $c_{0}\left(\alpha_{n}\right)=\left\{\left\{\beta_{n}\right\}: \beta_{n} \in \mathbf{C},\left\{\beta_{n} \alpha_{n}\right\} \in c_{0}\right\}$ with $\left\|\left\{\beta_{n}\right\}\right\|=\left\|\left\{\beta_{n} a_{n}\right\}\right\|_{\infty}$, then $\Psi: c_{0}\left(\alpha_{n}\right) \rightarrow Y:\left\{\beta_{n}\right\} \rightarrow \Sigma_{0}^{\infty} \beta_{n} y_{n}$ is a continuous linear isomorphism.

Proof. Since $y_{0}, y_{1}, \cdots, y_{n}$ are linearly independent, $y_{j}$ is not in the closed linear subspace $\mathbf{C} y_{0}+\cdots+\mathbf{C}_{j-1}$, and the projection from $\mathbf{C} y_{0}+\cdots$ : $+\mathbf{C} y_{j}$ to $\mathbf{C} y_{j}$ annihilating $\mathbf{C}_{0}+\cdots+\mathbf{C}_{j-1}$ is continuous with norm $K_{j}^{-1}$ (say) for $j=1,2, \cdots$, . Let $K_{0}=1$.

We now define the sequence $a_{n}$ inductively by $a_{0}=1$, and 


$$
a_{n} \geq 2^{n+1}\left\|y_{n}\right\| \cdot \prod_{j=1}^{n-1}\left[1+\frac{a_{j}}{\left\|y_{j}\right\| \cdot K_{j}}\right]
$$

for $n=1,2, \cdots$. . We shall apply the result when $\alpha_{n}$ is $n$ ! times the right-hand side, which we define to be $\gamma_{n}$ for future reference. The map $\Psi$ is now welldefined continuous and linear, because

$$
\left|\beta_{n}\right| \cdot\left\|y_{n}\right\| \leq\left\|\left\{\beta_{k}\right\}\right\| \cdot a_{n}^{-1}\left\|y_{n}\right\| \leq\left\|\left\{\beta_{k}\right\}\right\| \cdot 2^{-n-1}
$$

holds for $n=0,1,2, \cdots$, and all $\left\{\beta_{n}\right\}$ in $c_{0}\left(\alpha_{n}\right)$. Assume that $\left\{\beta_{n}\right\}$ is in $c_{0}\left(\alpha_{n}\right)$, and that $\Sigma_{0}^{\infty} \beta_{n} y_{n}=0$. Let $j$ be a nonnegative integer such that $\left|\beta_{j}\right| \alpha_{j}=$ $\left\|\left\{\beta_{n}\right\}\right\|=\sup \left\{\left|\beta_{n} \alpha_{n}\right|: n \geq 0\right\}$. Further $K_{j} \cdot\left|\beta_{j}\right| \cdot\left\|y_{j}\right\| \leq\left\|\Sigma_{n=0}^{j} \beta_{n} y_{n}\right\|$ by the definition of $K_{j}$. The triangle inequality and the choice of $j$ imply that

$$
K_{j}\left|\beta_{j}\right| \cdot\left\|y_{j}\right\| \leq \sum_{n=j+1}^{\infty}\left|\beta_{n}\right| \cdot\left\|y_{n}\right\| \leq \sum_{n=j+1}^{\infty}\left|\beta_{j}\right| \cdot \alpha_{j} \cdot\left\|y_{n}\right\| \cdot a_{n}^{-1} .
$$

By the choice of $\alpha_{n}$, we have

$$
a_{n} \geq 2^{n+1} \cdot\left\|y_{n}\right\| \cdot\left[1+a_{j} /\left\|y_{j}\right\| K\right]
$$

for $n \geq j+1$. Thus

$$
K_{j}\left|\beta_{j}\right| \cdot\left\|y_{j}\right\| \leq\left|\beta_{j}\right| \alpha_{j} \sum_{n=j+1}^{\infty} 2^{-n-1} \cdot \frac{\left\|y_{j}\right\| \cdot K_{j}}{a_{j}+\left\|y_{j}\right\| \cdot K_{j}}
$$

so that $\left|\beta_{j}\right| \leq 2^{-j-1} \cdot\left|\beta_{j}\right|$. This gives a contradiction unless $\left|\beta_{j}\right|=0$, and hence $\Psi$ is one-to-one.

Lemma 3.2. Let $\left\{a_{n}\right\}$ be a sequence of positive real numbers tending to infinity so that (sup $\left.\left\{a_{n} / a_{n+j}: n \geq 0\right\}\right)^{1 / j}$ tends to 0 as $j$ tends to infinity, and let $L$ be the left shift on $c_{0}\left(a_{n}\right)$. Then there is a nonnull torsion free L-divisible subspace of $c_{0}\left(a_{n}\right)$ that contains an infinite set of direct summands eacb of which is $\mathrm{C}[x]$-isomorpbic to $\mathrm{C}(x)$.

Proof. Since $L^{j}\left\{\beta_{n}\right\}=\left\{\beta_{n+j}\right\}$, we obtain $\left\|L^{j}\left\{\beta_{n}\right\}\right\|=\sup \left\{a_{n}\left|\beta_{n+j}\right|: n \geq 0\right\} \leq$ $\sup \left\{a_{n} / a_{n+j}: n \geq 0\right\} \cdot\left\|\left\{\beta_{n}\right\}\right\|$ for all $\left\{\beta_{n}\right\}$ in $c_{0}\left(a_{n}\right)$ so that $\left\|L^{j}\right\| \leq$ $\sup \left\{a_{n} / a_{n+j}: n \geq 0\right\}$ for $j=1,2, \cdots$. By the hypothesis on $\left\{a_{n}\right\}$, the linear operator $L$ is quasinilpotent. Let $W=c_{0}\left(a_{n}\right)$, and let $U=\bigcap_{1}^{\infty} L^{n} W$. Then $U$ contains the maximal $L$-divisible subspace of $W$, and $(\lambda I-L) U=U$ for all nonzero complex $\lambda$ because $L$ is quasinilpotent. If we show that $L U=U$ then $U$ will be the maximal $L$-divisible subspace of $W$. Just for the proof of this lemma 
we let $R$ denote the right shift on sequence spaces. If $x$ is in $L^{n+1} W$ with $x=$ $L^{n+1} y$, then $R x=(R L) L^{n} y$ so $R x$ and $L^{n} y$ differ by an element of the form $(\beta, 0,0, \cdots)$, and thus $R x$ is in $L^{n} W$. Because $U=\bigcap_{2}^{\infty} L^{n} W$, we have $R U \subseteq U$. Hence $U \subseteq L U$.

We now give $U$ the Fréchet topology induced by the norms $\|y\|_{n}=\left\|R^{n} y\right\|$ for all $y$ in $U$ and all positive intergers $n$. Then $L$ is continuous in this topology, and is not algebraic. By Lemma 1.1, the space $U$ contains an infinitely generated free $\mathrm{C}[x]$-submodule. Hence there are $z_{1}, z_{2}, \cdots$ in $U$ such that $\mathrm{C}[T] z_{1} \oplus$ $\mathrm{C}[T] z_{2} \oplus \cdots$ is contained in $U$, and each $\mathrm{C}[T] z_{j}$ is isomorphic to $\mathrm{C}[x]$ under the natural map.

Since a $\mathrm{C}[x]$-module is divisible if and only if is injective, $U$ is the maximal injective $\mathrm{C}[x]$-submodule of $W[2$, Proposition 5.1, p. 134]. The structure theory of injective $\mathrm{C}[x]$-modules implies that $U$ is a direct sum of injective $\mathrm{C}[x]$ submodules, some torsion free isomorphic to $\mathrm{C}(x)$, and the others are infinite torsion $\mathbf{C}[x]$-submodules (see [2] or $[7, \$ 12$, p. 37]). Because $L$ has a single eigenvector of ascent one, the only torsion $\mathrm{C}[x]$-submodule of $W$ is the set of all sequences each of which has only a finite number of nonzero terms. Suppose $U$ were a direct sum of only a finite number of submodules isomorphic to $\mathbf{C}(x)$ and the torsion submodule. Then each $z_{j}$ would be a sum $u_{j}+v_{j}$, where $u_{j}$ lies in the torsion submodule of $U$, and $v_{j}$ lies in the finite sum of $\mathbf{C}(x)$. Multiplying $z_{j}$ by a suitable nonzero polynomial $p_{j}$ gives $\bigoplus \mathrm{C}[T] p_{j}(T) z_{j}$ contained in the finite sum of the $\mathrm{C}(x)$. Hence $\left\{p_{j}(T) z_{j}: j=1,2, \cdots\right\}$ is a $\mathbf{C}(x)$-independent subset of a finite dimensional $\mathbf{C}(x)$ vector space giving a contradiction that proves the lemma.

Theorem 3.3. Let $Y$ be a Banacb space, and let $R$ be a continuous linear operator on $Y$. If $Y$ bas a nonnull $R$-divisible subspace, then $Y$ bas a nonnull torsion free $R$-divisible subspace.

Proof. Suppose that there is a nonzero $R$-torsion element $y$ in the $R$-divisible subspace of $Y$. Should there be no such element then this subspace is the required torsion free $R$-divisible subspace of $Y$. By replacing $y$ by $p(R) y$ for some suitable polynomial $p$ and $R$ by $R-\lambda I$ for some suitable $\lambda$ in the spectrum of $R$, we obtain a nonzero $y$ in an $R$-divisible subspace of $Y$ such that $R y=0$. Now normalize $y$ and $R$ so that $\|y\|=\|R\|=1$. Since $y$ is in an $R$-divisible subspace of $Y$, we may choose inductively $y_{0}, y_{1}, y_{2}, \ldots$ in this $R$-divisible subspace of $Y$ so that $y_{0}=y$ and $R y_{n+1}=y_{n}$. Now $y_{0}, y_{1}, y_{2}, \cdots$ are linearly independent because $R^{n} y_{n}=y_{0}$, which is nonzero, and $R^{n} y_{j}=0$ for $j=0,1, \cdots, n-1$. Otherwise the linear independence of $y_{0}, y_{1}, \ldots$ may be obtained from the structure theory of injective $\mathrm{C}[x]$-modules. We now apply Lemma 3.1 with 
$a_{n}=n ! \gamma_{n}$, where $\gamma_{n}$ is defined in Lemma 3.1. By the choice of $y_{0}, y_{1}, y_{2}, \cdots$ we have $R \Psi=\Psi L$, so that $\Psi$ is a $\mathrm{C}[x]$-monomorphism from $c_{0}\left(a_{n}\right)$ to $Y$ where $c_{0}\left(a_{n}\right)$ and $Y$ are regarded as $\mathrm{C}[x]$-modules via $L$ and $R$, respectively. The sequence $\left\{a_{n}\right\}$ satisfies the conditions of Lemma 3.2, because

$$
\frac{a_{n}}{a_{n+j}}=\frac{1}{2^{j}} \cdot \frac{\left\|y_{n}\right\|}{\left\|y_{n+j}\right\|} \frac{n !}{(n+j) !} \cdot \prod_{i=n}^{n+j-1}\left[1+\frac{a_{i}}{\left\|y_{i}\right\| \cdot K_{i}}\right]^{-1} \leq(j !)^{-1}
$$

since $R^{j} y_{n+j}=y_{n}$ and $\|R\|=1$. The $\mathrm{C}[x]$-monomorphism $\Psi$ carries the nonnull torsion free $L$-divisible subspace of $c_{0}\left(a_{n}\right)$ to a nonnull torsion free $R$-divisible subspace of $Y$. This completes the proof.

We now give a corollary of the proofs of Lemma 3.2 and Theorem 3.3. that leads to three natural questions, which we discuss after the corollary.

Corollary 3.4. Let $Y$ be a Banacb space, and let $R$ be a continuous linear operator on $Y$ with a nonzero divisible subspace. If $R$ is quasinilpotent or if $R$ bas an eigenvector in an $R$-divisible subspace of $Y$, then the maximal R-divisible subspace of $Y$ contains an infinite set of direct summands each of wbicb is $\mathrm{C}[x]$-isomorphic to $\mathrm{C}(x)$.

Proof. If $R$ has an eigenvector in an $R$-divisible subspace, then the proof of Theorem 3.3. and the full conclusion of Lemma 3.2 give this corollary. This leaves the case when $R$ is a one-to-one quasinilpotent operator. As in the proof of Lemma 3.3 the linear space $U=\bigcap_{1}^{\infty} R^{n} Y$ is the maximal $R$-divisible subspace of $Y$. The rest of the proof is similar to 3.3.

Three natural problems arise from the results in this section. Can the hypothesis " $R$ is quasinilpotent or $R$ has an eigenvalue in an $R$ divisible subspace of $Y$ " be omitted in Corollary 3.4? This would seem to require the construction of a one-to-one quasinilpotent operator from a norm on $\mathrm{C}(x)$ to correspond to Lemma 3.1 for a submodule of $Y$ that is $\mathrm{C}[x]$-isomorphic to $\mathrm{C}(x)$. One looks for a quasinilpotent operator so that the maximal divisible subspace may be turned into a Fréchet space so as to apply Lemma 1.1. Secondly can the infinite direct sum of modules isomorphic to $\mathrm{C}(x)$ be replaced by an uncountable direct sum in $1.1,3.1,3.3$, and 3.4? A related third question is: is there a submodule of our injective $\mathrm{C}[x]$-modules that is isomorphic to $\mathrm{C}[[x]]$, the $\mathrm{C}[x]$-module of all formal power series in $x$ (see [1])?

\section{REFERENCES}

1. G. R. All an, Embedding the algebra of formal power series in a Banach algebra, Proc. London Math. Soc. (3) 25 (1972), 329-340. 
2. H. Cartan and S. Eilenberg, Homological algebra, Princeton Univ. Press, Princeton, N. J., 1956. MR 17, 1040.

3. J. Dugundji, Topology, Allyn and Bacon, Boston, Mass., 1966. MR 33 \#1824.

4. B. E. Johnson, Continuity of linear operators commuting with continuous linear operators, Trans. Amer. Math. Soc. 128 (1967), 88-102. MR 35 \#4748.

5. - Continuity of operators commuting with quasi-nilpotent operators, Indiana Univ. Math. J. 20 (1971), 913-915.

6. B. E. Johnson and A. M. Sinclair, Continuity of linear operators commuting with continuous linear operators. II, Trans. Amer. Math. Soc. 146 (1969), 533-540. MR 40 \#4791.

7. I. Kaplansky, Infinite abelian groups, Univ. of Michigan Press, Ann Arbor, Mich., 1954. MR 16, 444.

DEPARTMENT OF MATHEMATICS, UNIVERSITY OF THE WITWATERSRAND, JOHANNESBURG, SOUTH AFRICA

Current address: Mathematical Institute, 20 Chambers Street, Edinburgh, EH1 1HZ, Scotland, Great Britain 